\title{
Evidence that the cAMP Pathway Controls Emergence of Both Primary and Appressorial Germ Tubes of Barley Powdery Mildew
}

\author{
Julia Kinane, ${ }^{1}$ Sussie Dalvin, ${ }^{1}$ Lene Bindslev, ${ }^{1}$ Alison Hall, ${ }^{2}$ Sarah Gurr, ${ }^{2}$ and Richard Oliver ${ }^{1}$ \\ ${ }^{1}$ Department of Physiology, Carlsberg Laboratory, Gamle Carlsberg Vej 10, DK-2500 Copenhagen Valby, \\ Denmark; '2Department of Plant Sciences, University of Oxford, South Parks Road, Oxford OX1 3RB, \\ U.K. \\ Accepted 10 January 2000.
}

\begin{abstract}
Development of conidia of barley powdery mildew involves the formation of a primary germ tube (PGT), an appressorial germ tube (AGT), and an appressorium. Previously, it was found that cyclic AMP (cAMP) was involved in these developmental processes. Comparison of development on the host surface with two types of cellulose membrane revealed that frequency of PGT emergence was surface independent. On one type of cellulose, where the frequencies of both AGT and appressorial differentiation were similar to that on the host surface, cAMP levels and protein kinase A (PKA) activities had a biphasic pattern with peaks at $15 \mathrm{~min}$ and $4 \mathrm{~h}$ after inoculation (prior to PGT and AGT emergence, respectively). The effect of manipulating cAMP levels was tested on another type of cellulose membrane, which stimulated a lower degree of AGT and appressorial formation than the host surface. Cholera toxin and forskolin, activators of adenylyl cyclase, significantly increased PGT emergence, but cAMP did not. Cholera toxin, forskolin, and cAMP increased the frequency of AGT and appressorial formation, but in a timedependent manner.
\end{abstract}

Conidia of the barley powdery mildew fungus, Blumeria (syn. Erysiphe) graminis (DC.) Speer f. sp. hordei, go through a distinct developmental pathway prior to penetration of barley epidermal cells. This involves formation of a primary germ tube (PGT) (germination), which is followed by the emergence of an appressorial germ tube (AGT) from the conidium. The AGT elongates, becomes septate, and swells at the tip to form a hook-shaped lobe (the appressorium). The appressorium is responsible for the penetration of the host (Carver et al. 1995). On a host surface, these morphological changes are highly synchronous. The signaling pathway(s) involved in these developmental processes have yet to be deciphered, although the involvement of cyclic AMP (cAMP) is implicated. Protein kinase A (PKA) transcripts and activities were identified in developing conidia, and exogenous cAMP and PKA inhibitors were found to affect germling development (Hall et al. 1999).

Corresponding author: R. P. Oliver; Telephone: +45 3327 5225; Fax: +45 3327 4766; E-mail: rpo@crc.dk
The classic cAMP signaling pathway involves a cell-surface receptor that responds to a specific extracellular stimulus and activates adenylyl cyclase (AC) (the enzyme that converts ATP to cAMP) via GTP-binding proteins. Changes in the activity of AC result in altered levels of cAMP. As a secondary messenger, cAMP mediates extracellular signals to PKA by binding to its regulatory subunits. The released and thereby activated catalytic subunits phosphorylate downstream targets that control the eventual phenotypic changes (Taylor et al. 1990).

AC and PKA have been identified and functionally analyzed in a number of phytopathogenic fungi and the cAMP signaling mechanism appears to play a critical role in the morphological differentiation associated with germination and infection. In Magnaporthe grisea, the causal agent of rice blast, AC null mutants showed a pleiotropic phenotype including reduced germination and an inability to form appressoria (Choi and Dean 1997). When similar experiments were carried out with the catalytic subunit of PKA, either the resulting mutants were unable to form appressoria (Mitchell and Dean 1995) or the appressorial formation was delayed (Xu et al. 1997). Moreover, the appressoria that were formed were smaller than those of the wild type and were unable to penetrate intact host tissue (Xu et al. 1997). In Ustilago maydis, the causal agent of corn smut, fungal dimorphism was altered in AC mutants while mutants defective in the regulatory subunit of PKA failed to form symptomatic galls in host plants and were defective in cytokinesis (Gold et al. 1994, 1997). In Neurospora crassa, mutants with reduced AC activity showed small, compact colonies, lacked extensive mycelial growth, and had tight clusters of dense conidia (Terenzi et al. 1976; Rosenberg and Pall 1979). These abnormalities were corrected in the presence of cAMP (Tenenzi et al. 1976) and by the introduction of a wild-type AC gene (Kore-Eda et al. 1991). Following both pharmacological studies (Yang and Dickman 1997), and the isolation, cloning, and characterization of the catalytic subunit of the PKA gene of Colletotrichum trifolii, Yang and Dickman (1999a, 1999b) have shown that PKA may be involved in germination and appressorial formation, but is more clearly involved in penetration of the host.

From such reports it is apparent that the cAMP signaling pathway controls the early development and pathogenesis of 
several phytopathogenic fungi in diverse ways. We set out to elucidate further the role of cAMP and PKA in germination and germling development in B. graminis f. sp. hordei. Mutational analysis has been pivotal to the study of cAMPdependent signaling pathways in other phytopathogenic fungi. Despite the power of this approach, the sequence of signal transduction events that underpin differentiation remains obscure. Cross-talk with other signal transduction pathways is a complicating factor particularly when dealing with genedisruption mutants (Krüger et al. 1998). B. graminis f. sp. hordei does not lend itself to mutational analysis due to its obligate biotrophic nature and the lack of any stable transformation system. Thus, pharmacological and biochemical analyses during germling development (up to appressorial formation) formed the core of our studies. Peaks in both cAMP level and PKA activity were observed prior to the emergence of both the PGT and the AGT. To determine whether these peaks were coincidental or causal to germ-tube emergence, cAMP levels were manipulated by feeding the conidia with activators of AC or with cAMP itself. The evidence suggests that fluxes in cAMP mediate the emergence of the two germ tubes but not differentiation of the appressorium.

\section{RESULTS}

\section{Germling development on barley epidermal cells} and two types of cellulose.

Different surfaces induce $B$. graminis f. sp. hordei development to the stage of appressorial formation to various degrees, ranging from the highly inductive host surface (barley epidermal cells) to non-inductive glass (Hall et al. 1999). Between these two extremes lie cellulose membranes, which themselves can be differentially inductive. Kobayshi et al. (1991) reported that PGT, AGT, and appressorial formation occurred on a cellulose membrane at rates similar to those on the host surface, whereas Hall et al. (1999) used a different kind of cellulose membrane that induced morphogenesis to a lesser degree.

While the barley epidermis is highly inductive to germling development, it poses technical difficulties in signal transduction studies. For example, in experiments in which germlings on the barley epidermis are treated with pharmacological agents, the epidermal cells may alter the chemical in some way or may prevent it from coming into contact with the germling. In addition, it is difficult to inoculate and harvest a large quantity of germlings in a short period of time on the epidermal cells. It was necessary to establish an experimental system that allowed the role of cAMP and PKA in germling development to be studied more easily. Therefore, two hydrated cellulose membranes, designated cellulose 1 and cellulose 2 and used by Kobayshi et al. (1991) and Hall et al. (1999), respectively, were used in these studies.

Initially, we compared germling development on the two cellulose membranes with that on the host surface. There was no significant interaction between any of the surfaces with respect to germination (Kruskal-Wallis interaction analysis, $H$ $=20.42 ; 28 \mathrm{df} ; P>0.05)$, indicating that the fungus performed similarly on all three surfaces (Fig. 1A). The maximum rate of germination occurred between 1 and $2 \mathrm{~h}$ after inoculation (hai). On all surfaces, 75 to $80 \%$ of the conidia germinated. With respect to formation of AGT and appressorial formation, there were significant interactions between the three surfaces, indicating differences in fungal development on these surfaces $(H=48.53$ and 44.95 for AGT and appressorial differentiation, respectively; $28 \mathrm{df} ; P<0.05$ ) (Fig. $1 \mathrm{~B}$ and $\mathrm{C}$ ). On cellu-
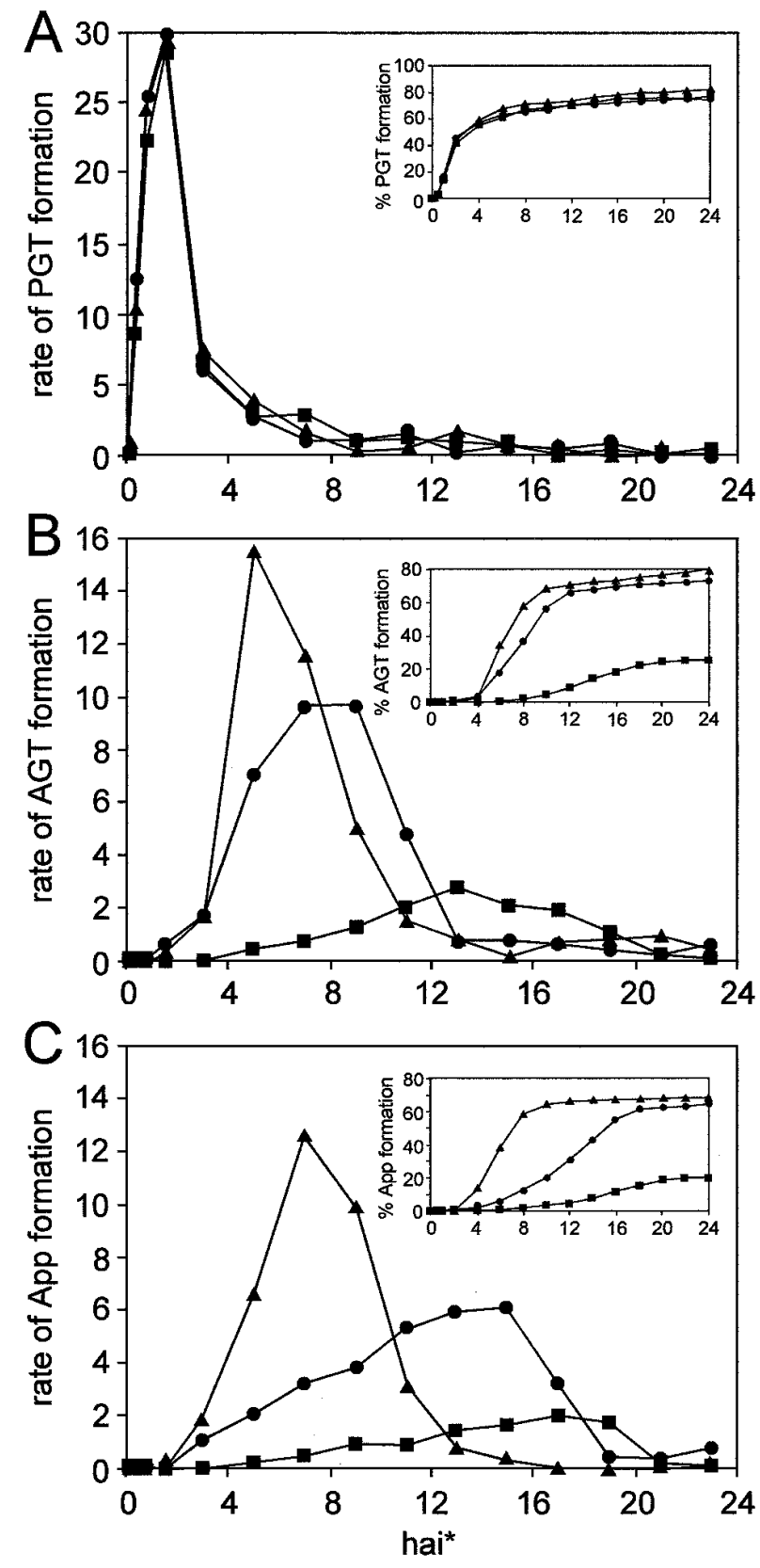

cellulose1-a-cellulose2- - barley epidermal cells

Fig. 1. Rate (number of spores reaching each stage of fungal development per hour) of germling development on barley epidermal cells and two types of cellulose. Inserts show frequencies of spores at each stage. Development was divided into (A) primary germ tube (PGT), (B) appressorial germ tube (AGT), and (C) appressorial (App) formation. On each surface, 400 conidia were scored, PGT formation was expressed as percentage of total number of conidia, and later stages were expressed as percentage of germinated conidia. Values shown are the medians of six replicates. $*$ hai $=$ hours after inoculation. 
lose 1 , the final proportion of germinated conidia that formed AGTs $(72 \%)$ was not significantly different from that on the barley epidermal strips (79\%). On cellulose 2, the number of germinated conidia that formed AGT (approximately 25\%) was significantly lower than that on both the barley epidermal strips and cellulose 1. Compared with those on epidermal strips, conidia on cellulose 2 took several hours longer to develop AGTs (25\%) (Fig. 1B).

The final number of germlings forming appressoria on cellulose $1(65 \%)$ was not significantly different from that on the barley epidermal strips (73\%). However, the conidia on cellulose 1 took up to $8 \mathrm{~h}$ longer than those on epidermal strips to develop appressoria (Fig. 2C). On cellulose 2, the number of germinated conidia that produced appressoria (approximately $20 \%$ ) was significantly lower than that on epidermal strips and it took these germlings up to $10 \mathrm{~h}$ longer to reach the appressorial stage. From these results, cellulose 1 was confirmed to be similar in inductive capacity to the host surface up to appressorial formation and therefore was used as a suitable surface to monitor cAMP levels and PKA activities during normal germling development. Cellulose 2 was confirmed to be a less stimulatory surface and thus was used to study the effect of pharmacological compounds postulated to stimulate development.

cAMP levels during germling development on cellulose 1.

To determine changes in cAMP levels during development, cAMP was measured in $B$. graminis f. sp. hordei conidia at the time of inoculation and $0.25,0.5,1,2,4,8,12,16$, and 20 hai. cAMP levels rose significantly (Kruskal-Wallis test; $H=$ 25.65; $P<0.05$ ) from 2 amol per spore at inoculation to 17 amol per spore 25 min later (Fig. 2). Subsequently, cAMP decreased to 5 amol per spore at 1 hai. This was followed by a second significant increase of cAMP to 39 amol per spore at 4 hai. By 8 hai, the levels of cAMP had dropped once more to

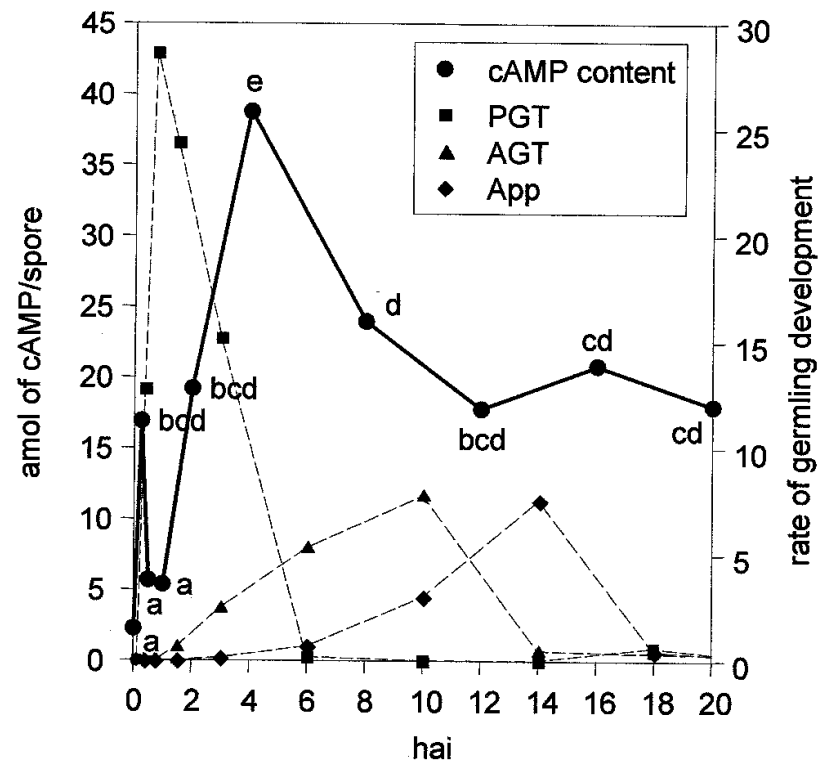

Fig. 2. Cyclic AMP (cAMP) levels in developing conidia on cellulose 1. Assessment of germling developmental stages described in Figure 1. Different letters $(\mathrm{a}, \mathrm{b}, \mathrm{c}, \mathrm{d}, \mathrm{e})$ indicate significant $(P=0.05)$ differences in cAMP levels with the Kruskal-Wallis test.
24 amol per spore, and did not significantly change between then and the end of monitoring (Fig. 2). Notably, the first peak in cAMP levels occurred prior to PGT emergence and the second prior to AGT emergence (Fig. 2). There were no significant changes in cAMP levels prior to or during appressorial formation. We attempted to monitor cAMP levels in developing germlings on the barley leaf surface. However, these results were unsatisfactory due to highly variable results in the control (uninoculated) leaves. Despite this, cAMP levels in germlings at 2 and 4 hai (just prior to and when the AGTs start to emerge) was significantly higher than the controls (data not shown). Interestingly, on glass, a surface where $B$. graminis $\mathrm{f}$. sp. hordei forms very few AGTs, there was no significant increase in cAMP levels at these times (Hall and Gurr 2000).

PKA activity during germling development on cellulose 1 .

Since cAMP activates PKA, the extractable PKA activities were measured during germling development. PKA activity followed a biphasic pattern that was similar to that shown by cAMP levels (Fig. 3). Within 15 min of conidial deposition on the cellulose membrane, the PKA activities significantly (Kruskal-Wallis test; $H=19.10 ; P<0.05$ ) increased from 9 $\mu \mathrm{U}$ per spore to $16 \mu \mathrm{U}$ per spore. Between $15 \mathrm{~min}$ and 1 hai, there was a significant decrease in PKA activity. After $1 \mathrm{~h}$, the activity of PKA increased to reach a peak of $19 \mu \mathrm{U}$ per spore at 4 hai. Between 4 and 8 hai, the PKA activity decreased, and between 8 and 20 hai, the PKA activity did not significantly change. The two peaks of PKA activity occurred prior to PGT and AGT emergence (Fig. 3). There was no significant change in PKA activity prior to or during appressorial formation.

\section{Effect of cholera toxin and forskolin (activators of AC) on the formation of PGT, AGT, and appressoria on cellulose 2.}

The changes in PKA and cAMP prior to both PGT and AGT emergence suggested that cAMP may mediate differentiation. To investigate this, conidia were inoculated onto segments of cellulose 2 laid over solutions of either cholera toxin

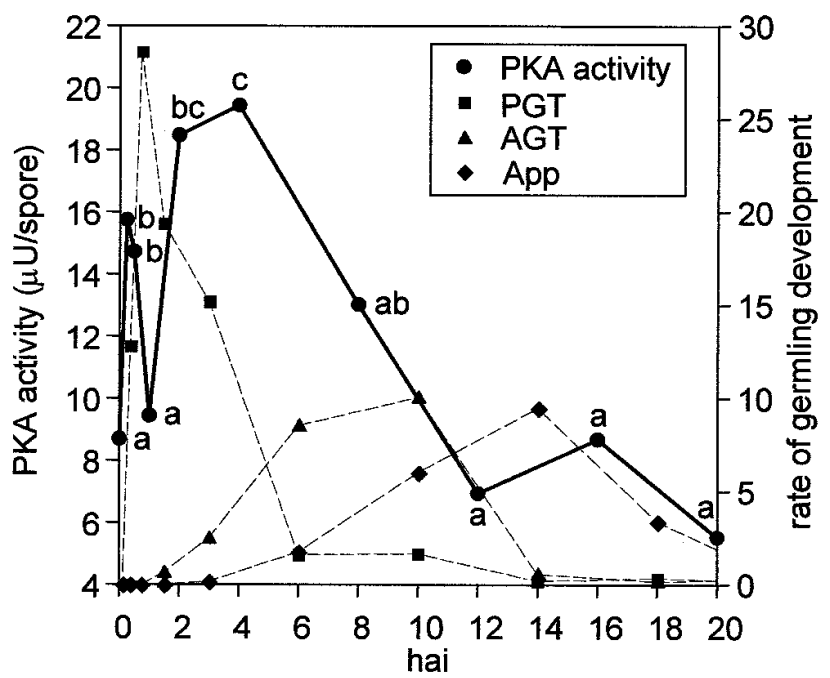

Fig. 3. Protein kinase A (PKA) activity of developing conidia on cellulose 1. Assessment of germling developmental stages described in Figure 1. Different letters $(\mathrm{a}, \mathrm{b}, \mathrm{c})$ indicate significant $(P=0.05)$ differences in PKA activities with the Kruskal-Wallis test. 
or forskolin (AC activators) or their control solutions. The pharmacological agents or control solutions were present either continuously for $24 \mathrm{~h}$ or from $0.5,1,2,4,6,8,10$, or 12 hai. In all cases, the conidia were assessed after $24 \mathrm{~h}$ for the presence of PGT, AGT, and appressoria. The results are presented in Figure 4A and B. Both cholera toxin and forskolin significantly increased germination, but only when added between $t=0$ and 4 hai. When cholera toxin or forskolin was added at any time after 4 hai, the germination frequency was not significantly higher than that of the controls. This suggests that ungerminated conidia lose their potential to germinate (or to be induced to germinate) after 4 hai. Conversely, cholera toxin and forskolin had no effect on the formation of AGTs or appressoria when applied at any time up to 1 hai. When applied any time between 2 and 8 hai, the frequency of AGT or appressorial formation was significantly higher than the control. The 1-h refractory period suggests that the conidia are incapable of receiving a stimulatory signal for AGT emer-
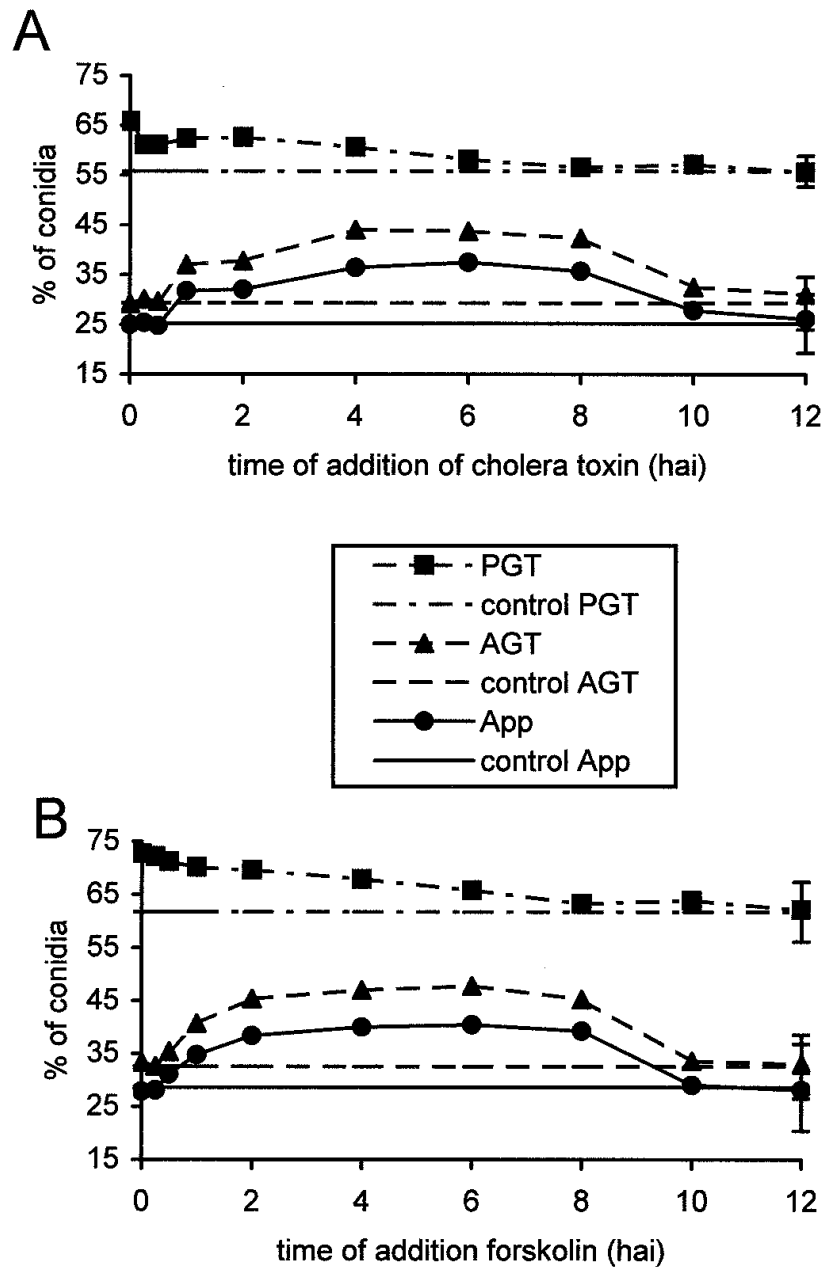

Fig. 4. Effect of adenylyl cyclase (AC) activators (A) cholera toxin and (B) forskolin on germling development on cellulose 2 membrane. Assessment of germling developmental stages described in Figure 1. Data were normalized with arc-sine transformation. Error bars indicating Tukey values $(P=0.05)$ following analysis of variance (ANOVA) are placed on the mean control value; any point outside the error bar is significantly $(P<0.05)$ different from the control. gence before 1 hai. However, when the AC activators were added at 10 or 12 hai, the numbers of AGT and appressoria were not significantly higher than those in the control. This result implies that germlings lose their ability to elongate an AGT and produce an appressorium (or receive the stimulus to do so) after 8 hai.

\section{Effect of exogenous cAMP on formation of PGT, AGT, and appressoria on cellulose 2 .}

The previous results suggested that temporal changes in cAMP levels are important for germling development. To assess the effects of exogenous application of cAMP on germling development, conidia were inoculated onto cellulose 2 laid over solutions of 1 or $10 \mu \mathrm{M}$ cAMP. The cAMP was present either continuously for $24 \mathrm{~h}$ or from $0.5,1,2,4,6,8,10$, or 12 hai. At 24 hai, the germlings were assessed for the pres-
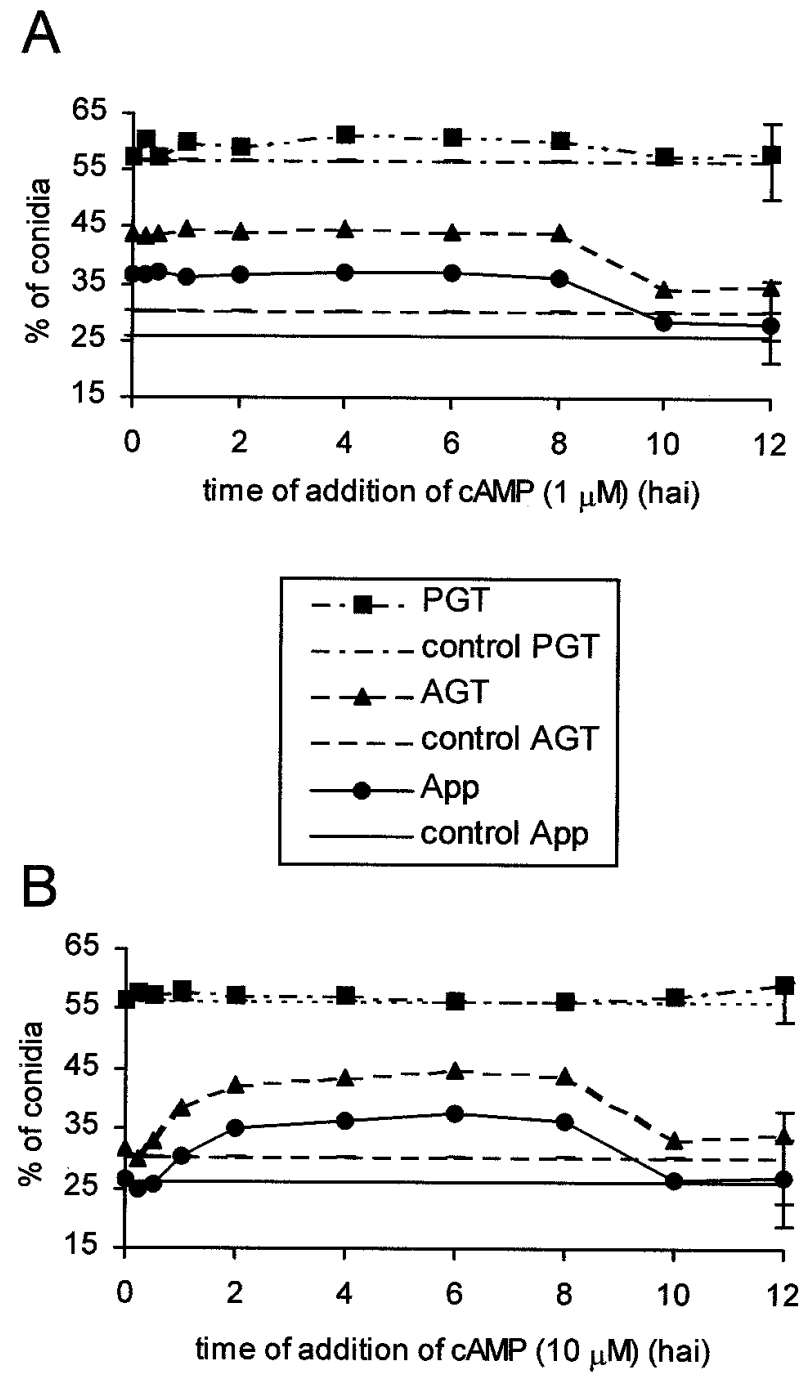

Fig. 5. Effect of exogenous cyclic AMP (cAMP) $(\mathbf{A}, 1 \mu \mathrm{M} ; \mathbf{B}, 10 \mu \mathrm{M})$ on germling development on cellulose 2 membrane. Assessment of germling developmental stages described in Figure 1. Data were normalized with arc-sine transformation. Error bars indicating Tukey values $(P=0.05)$ following analysis of variance (ANOVA) are placed on the mean control line; any point outside the error bar is significantly $(P<$ 0.05 ) different from the control. 
ence of PGT, AGT, and appressoria. Addition of cAMP (1 or $10 \mu \mathrm{M})$ had no significant effect on germination regardless of when it was added (Fig. 5A and B). This result was unexpected since activators of AC promoted germination. It suggests that exogenous cAMP cannot be efficiently taken up by the ungerminated conidium whereas AC activators can. The AC activators then increase endogenous cAMP levels (which subsequently affect germination).

If $1 \mu \mathrm{M}$ cAMP was added up to 8 hai, it significantly increased the numbers of AGTs and appressoria formed (Fig. 5A). Higher concentrations-10 $-10 \mathrm{M}$ (Fig. 5B) and $100 \mu \mathrm{M}$ (data not shown) - of cAMP had no effect on the formation of AGTs and appressoria if added up to 1 hai. However, the frequencies of AGT and appressorial formation significantly increased if cAMP was added between 2 and 8 hai (Fig. 5B). These differences in results at various concentrations of cAMP indicate two things. First, cAMP induces AGT and appressorial formation. Second, high levels of cAMP at or be-
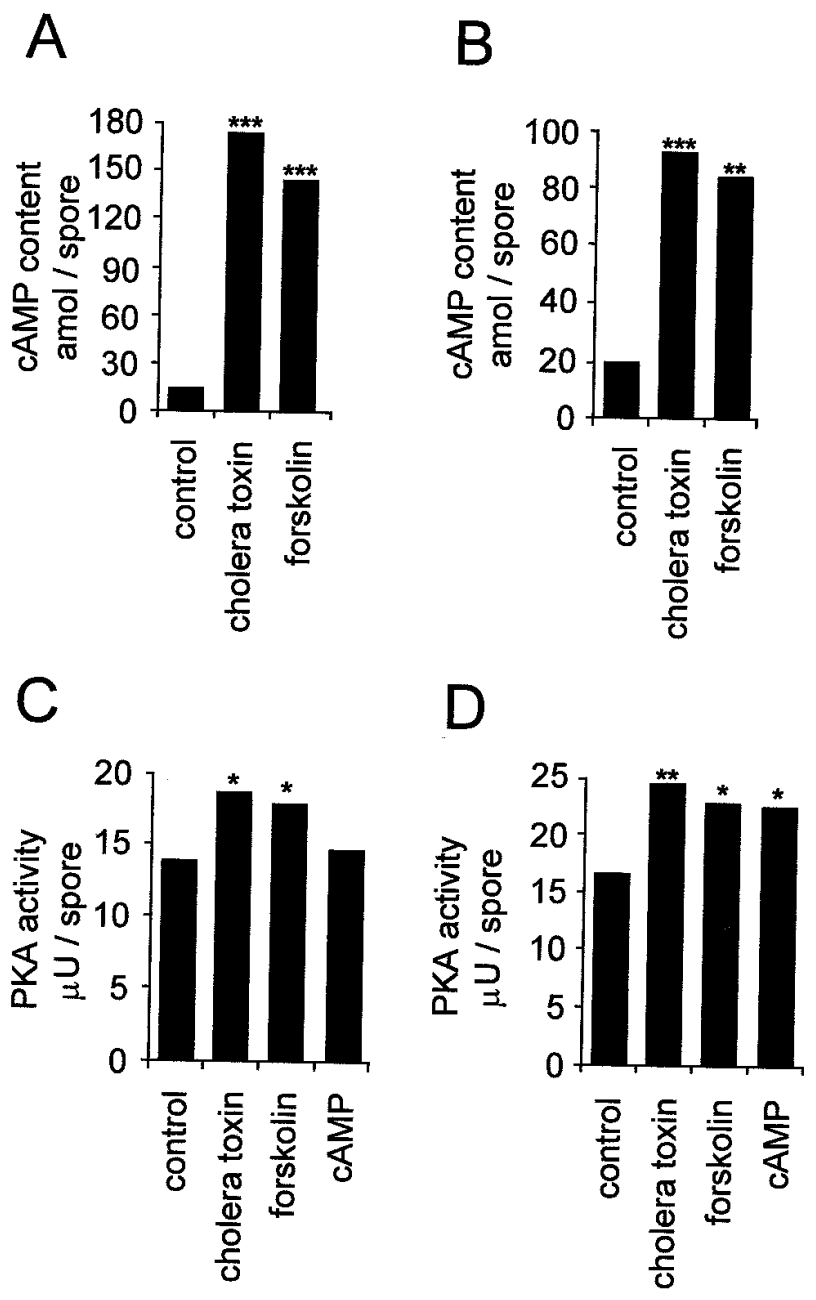

Fig. 6. Effect of (A, B) cholera toxin and forskolin on endogenous conidial cyclic AMP (cAMP) levels and (C, D) cholera toxin, forskolin, and cAMP on protein kinase A (PKA) activities on cellulose 2 membrane. Chemicals were added either $(\mathbf{A}, \mathbf{C})$ at inoculation or $(\mathbf{B}, \mathbf{D}) 3 \mathrm{~h}$ after inoculation, and were present for $15 \mathrm{~min}$. Three replicates were carried out for each time point. *,**, *** indicate significant difference from the mean control value at $P=0.05,0.01,0.001$, respectively, with Tukey's test following analysis of variance (ANOVA). fore 1 hai are not inductive to these stages. This suggests that cAMP should not be present in excessive amounts prior to 1 hai, if the frequencies of AGTs and appressoria are to be increased. Again, when added at 10 or 12 hai, cAMP (1 or 10 $\mu \mathrm{M})$ had no significant effect on the level of AGT and appressorial differentiation (Fig. 5A and B).

\section{Do AC activators or CAMP affect CAMP levels or PKA activities?}

We have shown (i) that cholera toxin, forskolin, and exogenous cAMP can promote germ-tube elongation and appressorial formation and (ii) that the timing of germ-tube formation correlates with fluxes in cAMP level and PKA activity. To link these phenomena, we investigated whether cholera toxin or forskolin can affect cAMP levels and PKA activities, and if exogenous cAMP $(1 \mu \mathrm{M})$ affects PKA activities. The compounds were supplied to the spores for $15 \mathrm{~min}$ either at inoculation $(t=0)$ or at 3 hai (when most of the spores have germinated), and PKA activities and cAMP levels were measured. When the AC activators were added at inoculation, significant $(P<0.05)$ increases in cAMP and PKA activities were observed (Fig. 6A and $\mathrm{C}$ ) (analysis of variance [ANOVA]; $F=311,2.6 \mathrm{df}, P<0.001$, and $F=5.59,3.8 \mathrm{df}, P$ $<0.05$; for cAMP levels and PKA activities. respectively). However. if cAMP was added at $t=0$ there was no increase in PKA activity (Fig. 6C). When the AC activators were added at 3 hai, significant increases in cAMP levels and PKA activities were again observed (ANOVA; $F=378,2.6 \mathrm{df}, P<$ 0.001 ; and $F=10 ; 3.8 \mathrm{df}, P<0.01$; for cAMP levels and PKA activities, respectively), presented in Figure $6 \mathrm{~B}$ and $\mathrm{D}$. There was also a significant increase in PKA activity when cAMP was added at this time (Fig. 6D). These results indicate that cholera toxin and forskolin increase conidial cAMP levels and PKA activities both before and after PGT emergence. However, exogenous cAMP only increased PKA activity after the PGT had emerged. This suggests that both cholera toxin and forskolin can either enter the spore or bind to a receptor on it before it has germinated and then increase endogenous cAMP levels, but that cAMP can only efficiently enter the germling after PGT emergence.

\section{DISCUSSION}

Following a recent report by Hall et al. (1999) that cAMP and PKA are involved in the early stages of $B$. graminis $\mathrm{f}$. $\mathrm{sp}$. hordei development (namely, PGT, AGT, and appressorial formation), we set out to determine their roles more precisely. To do this we monitored and manipulated cAMP levels and PKA activities during germling development.

\section{PGT formation.}

While the barley epidermis supports the most complete development of B. graminis f. sp. hordei, it poses technical difficulties with pharmacological and biochemical studies. Cellulose membranes are more amenable to such studies, and in this report two types were used. Germination rates on two different celluloses were similar to that on the host surface (epidermal barley cells) (Fig. 1). Other lines of evidence also suggest that germination of $B$. graminis $\mathrm{f}$. sp. hordei conidia is not surface dependent. Conidia germinate rapidly when held in a simulated airborne state on $0.5-\mu \mathrm{m}$ spider suspension 
threads (Carver and Ingerson 1987), or when floating on liquid surfaces (Gay et al. 1985; Nicholson et al. 1993). Germination is completely suppressed when the conidium is on a spore chain attached to the mother colony (Carver et al. 1995) but occurs rapidly following separation from it. Thus, the separation of the conidium from the spore chain may initiate germination. It may result from an increase in cAMP, since we found a rapid increase in cAMP level and in PKA activity in the first $15 \mathrm{~min}$ after inoculation onto cellulose (prior to PGT emergence) (Figs. 2 and 3). When cAMP levels were artificially increased with AC activators, germination was enhanced, suggesting that cAMP can mediate germination (Figs. 4 and 6). In $M$. grisea, there is evidence to suggest that cAMP is involved in germination. Deletion of an AC gene ( $m a c 1)$ resulted in a pleiotropic phenotype including delayed germination, while addition of monobutyryl-cAMP (a cAMP analogue) restored normal germination rates (Choi and Dean 1997). Changes in cAMP levels also appear to be associated with spore germination in other fungi. For example, in Mucor rouxii and Phycomyces blakesleeanus there is a rapid but transient increase in cAMP levels within 10 min of spores being transferred to conditions that induce germination (Dewerchin and Van Laere 1984; Van Mulders and Van Laere 1984; Tomes and Moreno 1990).

In this study, while AC activators increased germination, exogenous cAMP did not (Fig. 5). This was surprising since addition of AC activators or addition of cAMP would be expected to give the same result. We suggest that low concentrations of cAMP $(1$ or $10 \mu \mathrm{M})$ did not enter the spore in sufficient concentrations (to affect germination) prior to germination. The addition of cholera toxin and forskolin at time of inoculation increased cAMP levels and PKA activities, but addition of cAMP at this time did not alter PKA activity (Fig. 6). When Hall et al. (1999) applied high concentrations of cAMP ( $1 \mathrm{mM})$ to conidia on cellulose 2 , germination was inhibited, whereas lower concentrations (similar to those used in this study) had no affect on germination. Thus, perhaps this high concentration of cAMP did enter the spore, but had a nonspecific inhibitory effect on conidium germination. While forskolin and cholera toxin increased endogenous cAMP levels, the mechanism by which they did so was not investigated. Forskolin is a cell-permeable diterpene, which suggests that it may easily enter the spore. Cholera toxin is a very large protein (MW 84,000), with A and B polypeptide subunits. The B subunit is known to bind to a ganglioside receptor in the intestinal epithelium in humans and assists in the transport of the A subunit into the cells. It acts by the ADP ribosylation of the $\alpha$-subunit of a GTPbinding protein that then activates AC (Hepler and Gilman 1992). Cholera toxin has been shown to specifically bind to the cells of other fungi, for example Saccharomyces boulardii and S. cerevisiae (Brandao et al. 1998). St. Leger et al. (1989) demonstrated that the plasma membrane of Metarhizium anisopliae (a filamentous fungus that forms an appressorium) contains GTP binding proteins that bind to cholera toxin. The B subunit of cholera toxin may bind to a receptor on the developing $B$. graminis f. sp. hordei spore, allowing the A subunit to enter and activate AC. In preliminary work for this study, it was found that both subunits are required to have an effect on the fungus, and that denatured cholera toxin was ineffective.

\section{AGT formation.}

Germinating B. graminis f. sp. hordei conidia can respond to characteristics of the underlying surface. Perception of and response to these characteristics appear to be vital for growth and differentiation of the AGT (Carver and Ingerson 1987). Induction factors may include hydrophobicity of the underlying surface (Carver et al. 1996), cutin monomers (Francis et al. 1996) released from the host cuticle by fungal cutinases (Nicholson 1996; Nicholson and Kunoh 1995), and cellulose or its components that may be released by fungal cellulases (Suzuki et al. 1998). In this study, on cellulose 1, (the cellulose that induced the same frequency of conidia producing AGTs as the host surface), there was first an increase in cAMP levels and PKA activities (shortly after inoculation), followed by a decline, and subsequently by a second increase (Figs. 2 and 3). This second increase occurred prior to AGT emergence. We suggest that the second increase in cAMP level and PKA activity mediates AGT emergence. To test whether this was the case, activators of AC (cholera toxin or forskolin) or cAMP were added to cellulose 2 (the cellulose that induced relatively few conidia to produce AGTs) throughout development. In all cases, the frequencies of AGT formation increased. However, the timing of application of the chemical was critical. If low levels of cAMP $(1 \mu \mathrm{M})$ were added at any time during germling development, the number of germinated conidia forming AGTs increased by approximately $15 \%$ (Fig. 6). If either higher levels of cAMP (10 or $100 \mu \mathrm{M}$ ) or persistent activators of AC were added at the time of, or after, the second increase in cAMP and PKA on cellulose 1 (i.e., after 1 hai), the frequency of AGT formation also substantially increased (same magnitude as above). This increase in AGTs does not occur if the chemicals are added before 1 hai (Figs. 4 and 5). Thus, it appears that the cAMP pathway may indeed control emergence of AGTs. These results also indicate that it is essential to have a period of relatively low levels of cAMP followed by an increase, for induction of high frequencies of AGT formation.

While the above results indicate that cAMP triggers AGT emergence, the frequency of AGTs on cellulose 2 (40 to 50\%), even when treated with cAMP or AC activators, was never as high as on cellulose 1 or the barley epidermis (70 to $80 \%$ ). Perhaps cellulose 2 has an inhibitory effect downstream of cAMP that affects AGT emergence or possibly another signaling pathway is also involved in AGT formation.

\section{Appressorial formation.}

From our results, cAMP and PKA appear to control germtube emergence, but we have no evidence that they control appressorial formation. Increases in cAMP levels and PKA activities preceded germ-tube emergence but not appressorial differentiation. Also, addition of cAMP or AC stimulators did not increase the proportion of AGTs that went on to produce appressoria on cellulose 2 (60 to 75\%). In contrast, on cellulose 1 or on the barley epidermis, approximately $90 \%$ of the AGTs formed appressoria. This indicates that a separate stimulus may be involved in the formation of the appressorium. This is consistent with Carver and Ingerson's (1987) conclusions that formation of normal appressoria represents a stage in development separate from AGT formation. Presumably, the AGT responds to surface characteristic(s) and stimulates the differentiation of an appressorium. That the 
cAMP pathway does not mediate appressorial formation is in contrast to reports on $M$. grisea that suggest that cAMP regulates appressorium formation. Lee and Dean (1993) found that addition of exogenous cAMP to germinating conidia of $M$. grisea induced appressorium formation. Moreover, transformants lacking mac1 failed to produce appressoria. This abnormality was corrected in the presence of monobutyrylcAMP (Choi and Dean 1997).

In summary, we have examined the timing of events during development of $B$. graminis f. sp. hordei from germination to appressorial formation, and found evidence that, following release from the mother spore chain and subsequent deposition, there is a flux in conidial cAMP levels and PKA activities that mediates PGT emergence. We hypothesize that once the PGT has emerged, it perceives surface characteristics that stimulate AGT emergence via another flux in the cAMP and PKA pathway. The surface characteristic(s) that are perceived to stimulate the second increase in cAMP and AGT emergence are still unclear, as are the downstream effect(s) of the activated PKA.

\section{MATERIALS AND METHODS}

\section{Fungal and plant material.}

B. graminis f. sp. hordei race C15 (Kølster et al. 1986) was used in all experiments. Seeds of the barley cultivar Golden Promise were germinated in potting compost covered with vermiculite in a spore-proof growth cabinet, temperature $22 \pm$ $2{ }^{\circ} \mathrm{C}$ with a 18 -h photo period (sodium vapor lighting to give a light intensity of $150 \mu \mathrm{mol} \mathrm{s} \mathrm{s}^{-1} \mathrm{~m}^{-2}$ ). One day before conidia were required as experimental inoculum, infected leaves were shaken to remove older spores and ensure a supply of young ( $<24 \mathrm{~h}$ old) spores for experimentation. Seven-day-old seedlings were inoculated with conidia from 14-day-old colonies. Experimental inoculum was used from 7- to 11-day-old colonies. Inoculations were carried out in a settling tower, a device in which conidia are blown from a donor leaf and distributed evenly on the surface to be inoculated.

\section{Preparation and inoculation of host epidermis and cellulose membranes.}

The abaxial epidermis was removed from 7-day-old Golden Promise seedlings and floated (cuticle uppermost) on $2 \mathrm{ml}$ of Milli-Q (MQ) water in 19-mm-diameter petri dishes. Two cellulose membranes were used in these studies. Cellulose 1 was kindly supplied by $\mathrm{H}$. Kunoh and cellulose 2 (dialysis membrane, Cuprophan) was purchased from Medicell International (London). Segments (approximately $1 \mathrm{~cm}^{2}$ ) of the membrane were boiled in distilled water (three times for 30 min each, with 5-min washes between each boiling) dried, and stored in a desiccator at room temperature. Before use, the segments were rehydrated in MQ water, drained, and placed on $2 \%$ water agarose (Sigma, Vallenbaek Strand, Denmark) on a glass slide (which was previously washed with detergent, then rinsed with distilled water and finally with $96 \%$ ethanol) and placed in a laminar flow hood, where the membranes were allowed to dry.

The epidermal strips and membranes were inoculated to a known density (between 100 and 150 conidia per $\mathrm{mm}^{2}$ ) in a settling tower and then incubated at $100 \%$ relative humidity, $16 \pm 2{ }^{\circ} \mathrm{C}$ in darkness.

\section{Assessment of germling differentiation.}

Strips/membranes were either scored directly or fixed (strip/ membrane placed on Whatman filter paper moistened with 3:1 ethanol:glacial acetic acid [vol/vol] for 1 to $2 \mathrm{~min}$ at room temperature, then placed on filter paper [pre-wetted with distilled water], and stored at $4^{\circ} \mathrm{C}$ ) and viewed later. Conidia were viewed at $\times 200$ and scored for germination, formation of AGTs, and appressoria. On each surface, $4 \times 100$ conidia (from four different parts of the surface) were scored. Conidia touching other conidia were not considered. These values were averaged and treated as one replicate. Six replicates of each treatment were counted. Germination was defined as the production of a germ tube of length at least as great as its width. A germ tube was considered an AGT if its length was at least one-half the length of the spore. An AGT whose end was swollen (width at least twice the width of the germ tube at point of emergence from the conidium) and hooked was defined to have an appressorium.

\section{Preparation of material for cAMP and PKA assays.}

Segments of cellulose 1 membrane $(8 \times 8 \mathrm{~cm})$ were prepared as described above, placed on $2 \%$ agarose in square $(9 \times$ $9 \mathrm{~cm}$ ) petri dishes, allowed to dry, and inoculated to densities of 150 to 200 conidia per $\mathrm{mm}^{2}$. The entire inoculation procedure took less than $5 \mathrm{~min}$ in all cases. These membranes were either immediately harvested (time 0 ) or were incubated for up to $20 \mathrm{~h}$ before harvesting. To establish the developmental stage of the germlings at each time point, a small segment of membrane was placed alongside the test membrane and germling development of 400 spores was assessed. If the populations were not developing at the expected rate (this occurred rarely), the large membrane from that time point was discarded. Blank control membrane samples were included for each time point. The test and control membranes were harvested onto autoclaved aluminum foil and frozen immediately in liquid nitrogen for cAMP or PKA extraction. The membrane samples were removed from the foil and ground to a fine powder in a mortar (pre-cooled with liquid nitrogen) with acid-washed glass beads (75 to $150 \mu \mathrm{m}$, Sigma) in liquid nitrogen.

\section{cAMP extraction and assay.}

Three milliliters of $6 \%$ TCA was added to the powdered membrane segments, and the mixture was incubated on ice for $20 \mathrm{~min}$ and centrifuged at $1,300 \times \mathrm{g}, 4^{\circ} \mathrm{C}$ for $10 \mathrm{~min}$. The supernatant was removed and extracted with water-saturated ether $(5 \times \mathrm{vol} / \mathrm{vol})$. The sample was dried at $60^{\circ} \mathrm{C}$ under flowing nitrogen for approximately $1.5 \mathrm{~h}$ and stored at $-20^{\circ} \mathrm{C}$. cAMP was quantified by the cAMP enzyme immunoassay (EIA) system (Amersham, Birkerød, Denmark) according to the manufacturer's instructions. cAMP levels were converted to amol of cAMP per spore. cAMP was extracted from four replicate membrane segments per time point, and the cAMP assay was carried out in duplicate for each sample.

\section{PKA extraction and assay.}

Three milliliters of extraction buffer (10 mM Tris, $\mathrm{pH} 7.5$, $10 \mathrm{mM} \mathrm{MgCl}_{2}$ ) containing phenylmethylsulfonyl fluoride (1 $\mathrm{mM}$ ) was added to the powdered spores and membrane, mixed, and incubated on ice for $1 \mathrm{~h}$ (as modified from Yang and Dickman 1997). The samples were centrifuged $(3,000 \times$ 
$g$ ) at $4{ }^{\circ} \mathrm{C}$ for $10 \mathrm{~min}$ and $2.5 \mathrm{ml}$ of supernatant was passed through a PD-10 column (Amersham, Birkerød, Denmark) and eluted with $3.5 \mathrm{ml}$ of extraction buffer. The 3.5-ml elution was concentrated in a 4-ml Vivaspin concentrator (MWCO = 10,000) (Vivascience) to approximately $300 \mu \mathrm{l}$. The extracted PKA samples were stored at $-80^{\circ} \mathrm{C}$. The samples were standardized to $0.06 \mu \mathrm{g}$ of protein per $\mu \mathrm{l}$ with a protein assay kit (Bio-Rad, Hercules, CA) with bovine serum albumin as a standard. The PKA activity assay (with no exogenous cAMP added) was performed with a nonradioactive PKA assay kit (PepTagA; Promega, Madison, WI) following the protocol provided with the kit, except that half the recommended amount of Kemptide was used. For each sample, $0.8 \mu \mathrm{g}$ of protein was used in the PKA assay. Phosphorylated Kemptide was separated from nonphosphorylated Kemptide on an $0.8 \%$ agarose gel (in $50 \mathrm{nM}$ Tris, $\mathrm{pH}$ 7.5) and the percentage of phosphorylated Kemptide was determined with a STORM 860 imaging system (Molecular Dynamics, Sunnyvale, CA). Percentage of phosphorylation was converted to standard units based on a standard curve correlating measurements from the STORM imaging system (excitation $560 \mathrm{~nm}$, emission 610 $\mathrm{nm}$ ) of known Kemptide concentrations. PKA activities were converted to $\mu \mathrm{U}$ per spore $(\mathrm{U}=\mathrm{nmol}$ of phosphate transferred to substrate $\min ^{-1} \mathrm{ml}^{-1}$ ). At each time point, PKA was extracted from four replicates, and the phosphorylation assay was carried out in triplicate for each sample.

\section{Pharmacological analysis.}

These studies involved the assessment of germling development after treatment with the pharmacological agents cAMP (1 and $10 \mu \mathrm{M}$ in MQ water) (Sigma), cholera toxin (5 ng per ml of MQ water) (Calbiochem, Darmstadt, Germany), or forskolin $(10 \mu \mathrm{M}$ in $0.1 \%$ ethanol; Calbiochem) at given time points after inoculation. The concentrations of AC activators used in these studies were the lowest required to give a significant effect on germination (determined from preliminary experiments). Squares $\left(1 \mathrm{~cm}^{2}\right)$ of cellulose 2 membrane were placed on $20-\mu$ d droplets of the pharmacological agent or its control solution aliquoted onto an agarose-covered glass slide (described above), dried, and inoculated in a settling tower to densities of between 150 and 200 conidia per $\mathrm{mm}^{2}$. The cellulose 2 membrane segment was either incubated for $24 \mathrm{~h}$ under the conditions described above, or at $0.25,0.5,1$, $2,4,6,8,10$, or 12 hai it was moved from a droplet of the control solution (either water or $0.1 \%$ ethanol) and laid onto a $20-\mu l$ droplet of the pharmacological agent and then incubated for the remainder of the $24 \mathrm{~h}$. Control experiments involved the transfer of cellulose 2 membrane from one control droplet to another at the same time points. Four replicates of each treatment were carried out. At 24 hai, the samples were either viewed immediately or fixed and scored as described above. The control results were very consistent, so their values were averaged and results from the pharmacological treated germlings were compared with that average.

\section{Effect of AC activators and CAMP on cAMP levels and PKA activity.}

cAMP levels and PKA activities of spores were assessed (15 min) following application of cholera toxin, forskolin, or cAMP at two time points, $t=0$ and 3 hai. Three replicates were carried out for each time point. For the first time point $(t$
$=0)$, cellulose 2 membranes $\left(64 \mathrm{~cm}^{2}\right)$, prepared as previously described, were placed on $2 \%$ agarose in square $\left(9 \times 9 \mathrm{~cm}^{2}\right)$ petri dishes, allowed to dry, and moved onto fresh agarose wetted with $1 \mathrm{ml}$ of cholera toxin $(5 \mathrm{ng} / \mathrm{ml})$, forskolin (10 $\mu \mathrm{M})$, cAMP $(1 \mu \mathrm{M})$, or their control solutions and inoculated. After $15 \mathrm{~min}$, the membranes were harvested and cAMP or PKA was extracted and assayed as above. For the second time point, 3 hai, $64-\mathrm{cm}^{2}$ cellulose 1 membranes were prepared and inoculated. Three hai, the conidia were viewed briefly at $\times 200$ to ensure high frequencies of germination. The membranes were moved onto agarose in other petri dishes that was wetted with $1 \mathrm{ml}$ of the pharmacological agents or the control solutions. After $15 \mathrm{~min}$, the membranes were harvested and cAMP or PKA was extracted and assayed.

\section{Experimental design and statistical analysis.}

All experiments were set up in a in a completely randomized design. The data approximated either to normal or nonnormal frequency distributions. For multiple comparisons, parametric ANOVA followed by the Tukey test was conducted on normal data. When the data approximated to non-normal frequency distributions, either the nonparametric KruskalWallis test was carried out or, following normalization (with arc-sine transformation), parametric ANOVA was conducted with the Tukey test. Results presented here are from a single experiment. The cAMP and PKA assays were performed twice and results between experiments were very consistent. All other experiments were conducted at least three times and there was no statistical difference between the experiments.

\section{ACKNOWLEDGMENTS}

We would like to thank H. Kunoh, Laboratory of Plant Pathology, Faculty of Bioresources, University, Tsu-city, 514, Japan, for the gift of cellulose 1. J. K. is funded by the E.U. TMR Cerepat Network grant FMRX-CT98-0241 to R. O., and A. H. by a BBSRC CASE award with Rhone-Poulenc.

\section{LITERATURE CITED}

Brandao, R. L., Castro, I. M., Bambirra, E. A., Amaral, S. C., Fietto, L. G., Tropia, M. J. M., Neves, M. J., dos Santos, R. G., Gomes, N. C. M., and Nicoli, J. R. 1998. Intracellular signal triggered by cholera toxin in Saccharomyces boulardii and Saccharomyces cerevisiae. Appl. Environ. Microbiol. 64:564-568.

Carver, T. L. W., and Ingerson, S. M. 1987. Responses of Erysiphe graminis germlings to contact with artificial and host surfaces. Physiol. Mol. Plant Pathol. 30:359-372.

Carver, T. L. W., Ingerson, S. M., and Thomas, B. J. 1996. Influences of host surface features on development of Erysiphe graminis and Erysiphe pisi. Pages 255-266 in: Plant Cuticles. G. Kerstiens, ed. BIOS Scientific Publishers, Oxford.

Carver, T. L. W., Ingerson-Morris, S. M., Thomas, B. J., and Zeyen, R. J. 1995. Early interactions during powdery mildew infection. Can. J. Bot. 73(suppl. 1):S632-S639.

Choi, W., and Dean, R. A. 1997. The adenylate cyclase gene MAC1 of Magnaporthe grisea controls appressorium formation and other aspects of growth and development. Plant Cell 9:1973-1983.

Dewerchin, M., and Van Laere, A. 1984. Trehalase activity and cAMP content during early development of Mucor rouxii spores. J. Bacteriol. 158:575-579.

Francis, S. A., Dewey, F. M., and Gurr, S. J. 1996. The role of cutinase in germling development and infection by Erysiphe graminis $\mathrm{f}$. sp. hordei. Physiol. Mol. Plant Pathol. 49:201-211.

Gay, J. K., Martin, M., and Ball, E. 1985. The impermeability of powdery mildew conidia and their germination in arid environments. Plant 
Pathol. 34:353-362.

Gold, S., Duncan, G., Barrett, K., and Kronstad, J. 1994. cAMP regulates morphogenesis in the fungal pathogen Ustilago maydis. Genes Dev. 8:2805-2816.

Gold, S. E., Brogdon, S. M., Mayorga, M. E., and Kronstad, J. W. 1997. The Ustilago maydis regulatory subunit of a cAMP-dependent protein kinase is required for gall formation in maize. Plant Cell 9:1585-1594.

Hall, A. A., Bindslev, L., Rouster, J., Rasmussen, S. W., Oliver, R. P., and Gurr, S. J. 1999. The involvement of cAMP and protein kinase A in conidial differentiation by Erysiphe graminis f. sp. hordei. Mol. Plant-Microbe Interact. 12:960-968.

Hall, A. A., and Gurr, S. J. 2000. Initiation of appressorial germ tube differentiation and appressorial hooking: Distinct morphological events regulated by cAMP signalling in Blumeria graminis f. sp. hordei. Physiol. Mol. Plant Pathol. 56:39-46.

Hepler, J. R., and Gilman, A. G. 1992. G proteins. Trends Biochem. Sci. 17:383-387.

Kobayshi, I., Tanaka, C., Yamoka, N., and Kunoh, H. 1991. Morphogenesis of Erysiphe graminis conidia on artificial membranes. Trans. Mycol. Soc. Jpn. 32:187-198.

Kore-Eda, S., Murayama, T., and Uno, I. 1991. Isolation and characterization of the adenylate cyclase structural gene of Neurospora crassa. Jpn. J. Genet. 66:317-334.

Kølster, P., Munk, L., Stølen, O., and Løhde, J. 1986. Near-isogenic barley lines with genes for resistance to powdery mildew. Crop Sc. 26: 903-907.

Krüger, J., Loubradou, G., Regenfelder, E., Hartmann, A., and Kahmann, R. 1998. Crosstalk between cAMP and pheromone signalling pathways in Ustilago maydis. Mol. Gen. Genet. 260:193-198.

Lee, Y. H., and Dean, R. A. 1993. cAMP regulates infection structure formation in the plant pathogenic fungus Magnaporthe grisea. Plant Cell 5:693-700.

Mitchell, T. K., and Dean, R. A. 1995. The cAMP-dependent protein kinase catalytic subunit is required for appressorium formation and pathogenesis by the rice blast pathogen Magnaporthe grisea. Plant Cell 7:1869-1878.

Nicholson, R. L. 1996. Adhesion of fungal propagules: Significance to the success of the fungal infection process. Pages 117-134 in: Histology, Ultrastructure and Molecular Cytology of Plant-Microorganism Interactions. M. Nicole and V. Gianinazzi, eds. Kluwer, Dordrecht, The Netherlands.

Nicholson, R. L., and Kunoh, H. 1995. Early interactions during pow- dery mildew infection. Can. J. Bot. 73(suppl. 1):S609-S615.

Nicholson, R. L., Kunoh, H., Shiraishi, T., and Yamada, T. 1993. Initiation of the infection process by Erysiphe graminis: Conversion of the conidial surface from hydrophobicity to hydrophilicity and influence of the conidial exudate in the hydrophobicity of the barley leaf surface. Physiol. Mol. Plant Pathol. 43:307-318.

Rosenberg, G., and Pall, M. L. 1979. Properties of two cyclic nucleotidedeficient mutants of Neuropsora crassa. J. Bacteriol. 137:1140-1144.

St. Leger, R. J., Roberts, D. W., and Staples, R. C. 1989. Novel GTPbinding proteins in plasma membranes of the fungus Metarhizium anisopliae. Biochem. Biophys. Res. Commun. 164:562-566.

Suzuki, S., Komiya, Y., Mitsui, T., Tsuyumu, S., Kunoh, H., Carver, T. L. W., and Nicholson, R. L. 1998. Release of cell wall degrading enzymes from conidia of Blumeria graminis on artificial substrata. Ann. Phytopathol. Soc. Jpn. 64:160-167.

Taylor, S. S., Buechler J. A., and Yonemoto, W. 1990. cAMP-dependent protein kinase: Framework for a diverse family of regulatory enzymes. Annu. Rev. Biochem. 59:971-1005.

Terenzi, H. F., Flawia, M. M., Tellez-Inon, M. T., and Torres, H. N. 1976. Control of Neuropsora crassa morphology by cyclic adenosine 3',5'-monophosphate and dibutyryl cyclic adenosine 3',5'-monophosphate. J. Bacteriol. 126:91-99.

Tomes, C., and Moreno, S. 1990. Phosphodiesterase activity and cyclic AMP content during early germination of Mucur rouxii spores. Exp. Mycol. 14:78-83.

Van Mulders, R. H., and Van Laere, A. J. 1984. Cyclic AMP, trehalase and germination of Phycomyces blakesleeanus spores. J. Gen. Microbiol. 130:541-547.

Xu, J.-R., Urban, M., Sweigard, J. A., and Hamer, J. E. 1997. The CPKA gene of Magnaporthe grisea is essential for appressorial penetration. Mol. Plant-Microbe Interact. 10:187-194.

Yang, Z., and Dickman, M. B. 1997. Regulation of cAMP and cAMP dependent protein kinase during conidial germination and appressorium formation in Colletotrichum trifolii. Physiol. Mol. Plant Pathol. 50:117-127.

Yang, Z., and Dickman, M. B. 1999a. Colletotrichum trifolii mutants disrupted in the catalytic subunit of cAMP-dependent protein kinase are nonpathogenic. Mol. Plant-Microbe Interact. 12:430-439.

Yang, Z., and Dickman, M. B. 1999b. Molecular cloning and characterization of $C t-P K A R$, a gene encoding the regulatory subunit of cAMPdependent protein kinase in Colletotrichum trifolii. Arch. Microbiol. 171:249-256 